\title{
Demonstration of a wideband submillimeter-wave low-noise receiver with 4-21 GHz IF output digitized by a high-speed 32 GSps ADC
}

\author{
T. Kojima ${ }^{1,2}$, H. Kiuchi ${ }^{1}$, K. Uemizu ${ }^{1}$, Y. Uzawa ${ }^{1,2}$, M. Kroug ${ }^{3}$, A. Gonzalez ${ }^{1,2}$, T. Dippon ${ }^{4}$, and T. Kageura ${ }^{4,5}$ \\ ${ }^{1}$ National Astronomical Observatory of Japan (NAOJ), Osawa 2-21-1, Mitaka, Tokyo, Japan \\ e-mail: t.kojima@nao.ac.jp \\ 2 Graduate University for Advanced Studies (SOKENDAI), Mitaka, Tokyo 181-8588, Japan \\ 3 The University of British Columbia, Vancouver, BC V6T 1Z4, Canada \\ ${ }^{4}$ Keysight Technologies Deutschland GmbH, Böblingen, Baden-Württemberg 71034, Germany \\ 5 Keysight Technologies Japan K.K., Hachioji, Tokyo 192-8550, Japan
}

Received 22 June 2020 / Accepted 13 July 2020

\begin{abstract}
We report on a $275-500 \mathrm{GHz}$ heterodyne receiver system in combination with a wideband intermediate-frequency (IF) backend to realize $17 \mathrm{GHz}$ instantaneous bandwidth. The receiver frontend implements a heterodyne mixer module that integrates a superconductorinsulator-superconductor (SIS) mixer chip and a cryogenic low-noise preamplifier. The SIS mixer is developed based on high-currentdensity junction technologies to achieve a wideband radio frequency (RF) and IF bandwidth. The IF backend comprises an IF chain divided into two channels for $4.0-11.5 \mathrm{GHz}$ and $11.3-21.0 \mathrm{GHz}$ and an analog-to-digital converter (ADC) module that is capable of high-speed sampling at 32 Giga samples per second with $12.5 \mathrm{GHz}$ bandwidth per channel and an effective number of bits of 6.5. The IF backend allows us to simultaneously cover the full 4-21 GHz IF range of the receiver frontend. The measured noise temperature of the receiver frontend was below three times the quantum noise $\left(h f / k_{\mathrm{B}}\right)$ over the entire RF band. A dual-polarization sideband-separating receiver based on this technique could provide up to $64 \mathrm{GHz}$ of instantaneous bandwidth, which demonstrates the possibility of future wideband radio astronomical observations with advanced submillimeter-wave heterodyne receivers.
\end{abstract}

Key words. instrumentation: detectors - techniques: spectroscopic

\section{Introduction}

High-sensitivity ultra-wideband coherent heterodyne receivers are highly demanded by the radio astronomy community and considered one of the highest priorities in development plans of major radio astronomy facilities. Receivers with a large instantaneous bandwidth can affect a variety of science cases. They can make it possible to conduct deep continuum surveys with the highest sensitivity, or blind redshift surveys and chemical spectral scans with fewer local oscillator (LO) frequency tunings, among others (ALMA Development Working Group 2018). Increasing the radio frequency (RF) bandwidth reduces the total number of receivers, which contributes to improving operability and maintainability and returns time used for those activities to science, as well as to conducting scientific observations with a seamless frequency scan, which reduces inaccuracies associated with multiple calibrations. The merits and capabilities of affecting science fields of wideband receivers can be maximized when this is combined with the high angular resolution and high sensitivity of radio interferometers, such as the Atacama Large Millimeter/submillimeter Array (ALMA) ${ }^{1}$.

Coherent receivers for millimeter and submillimeter wave astronomy are based on superconducting mixers because their

\footnotetext{
http://www . almaobservatory.org/
}

low noise temperature approaches physical limits, the quantum limit. The received RF signal is multiplied with a locally generated LO power and down-converted to intermediate frequency (IF) signals in the microwave range. Several studies in recent years have aimed to increase the current limits of RF or IF bandwidth. Hot-electron bolometer (HEB) mixers are promising devices to cover wide RF bandwidths at frequencies above $1 \mathrm{THz}$. The limitations in RF bandwidth are related to the signal-coupling scheme using lenses or waveguide components, and not to the HEB mixer itself. However, it is in principle difficult to achieve IF bandwidths greater than $10 \mathrm{GHz}$ because the mixing mechanism is fundamentally limited (Novoselov \& Cherednichenko 2017). On the other hand, receivers based on superconductor-insulatorsuperconductor (SIS) mixers at submillimeter wavelengths, up to about $1 \mathrm{THz}$, have attained quantum-limited low-noise performance with an RF fractional bandwidth of 20-30\%, and IF 4-8 GHz (Billade et al. 2013; Kerr \& Pan 2014; Mahieu et al. 2012; Tamura et al. 2015), or 4-12 GHz (Baryshev et al. 2015; Uzawa et al.2015). In recent years, several studies have attempted to increase the RF (Kojima et al. 2017) and IF (Tong 2015; Kojima et al. 2018) bandwidth of SIS mixers independently. This work has proven very challenging in terms of maintaining current advanced noise-temperature performance because there are some fundamental trade-offs in terms of bandwidth and 


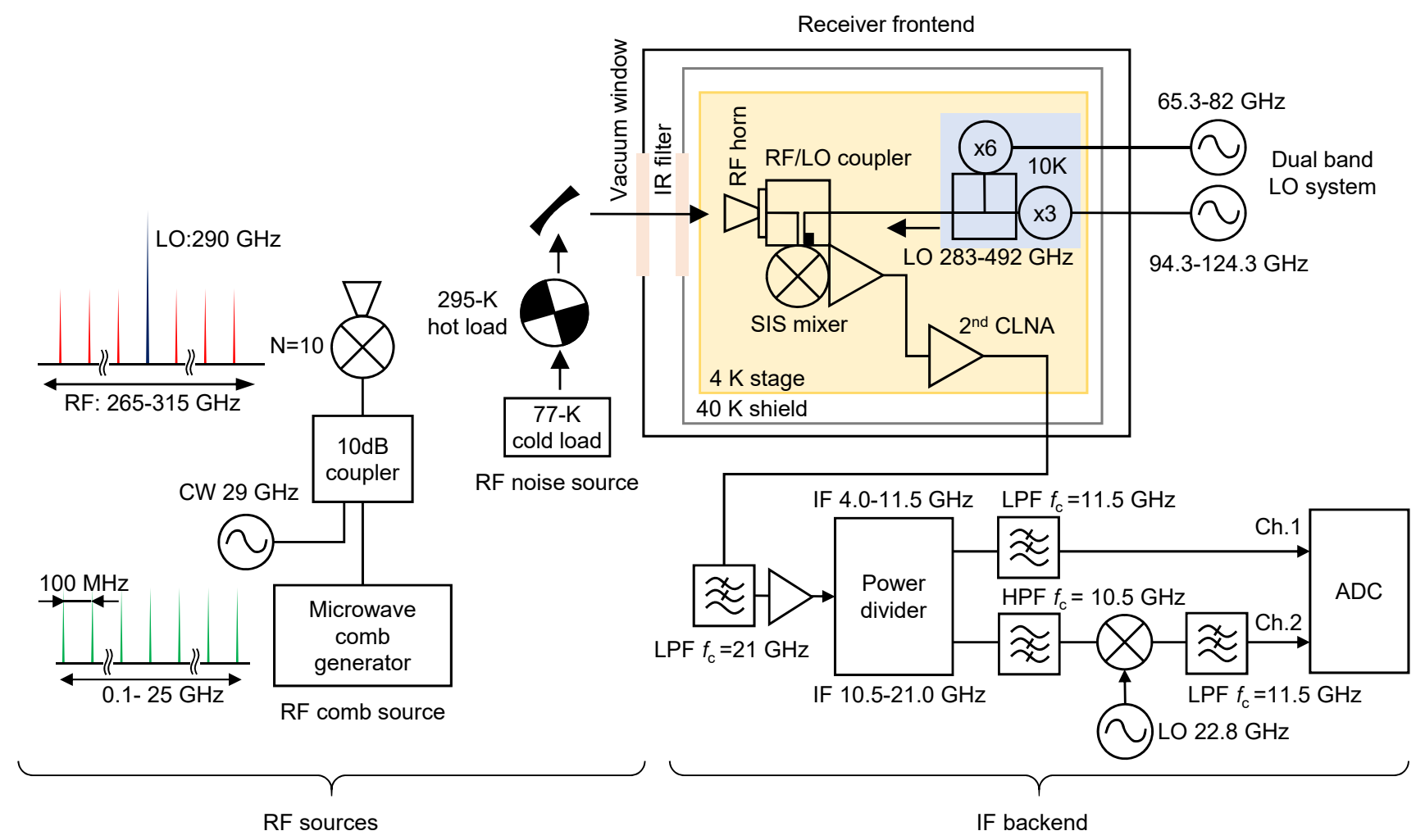

Fig. 1. Full receiver system developed in this study. The system consists of an RF source, receiver frontend, dual-band LO system, and IF backend. The RF noise source with blackbody loads at $295 \mathrm{~K}$ and $77 \mathrm{~K}$ is used for $Y$-factor measurement, while the RF comb source is used for verification of connectivity between two channels in the IF backend.

performance of the mixing elements. This is further exacerbated by the need to increase the bandwidth of all receiver subsystems surrounding the mixer and downstream: RF components, LO chain, analog IF circuits, and IF backend.

In this paper, we report on a double-sideband (DSB) receiver system that covers an RF range of $275-500 \mathrm{GHz}$ and IF range of $4-21 \mathrm{GHz}$ and achieves most advanced noise-temperature performance. It integrates wideband RF components, an SISmixer-preamplifier module, an LO system consisting of two lownoise continuous-wave (CW) sources, and an IF backend using a high-speed analog-to-digital converter (ADC) with 32 Giga samples per second (Gsps) and $12.5 \mathrm{GHz}$ bandwidth per channel performance. The fractional RF bandwidth of the receiver slightly exceeds $60 \%$, and is limited by the frequency range of single-mode propagation in WR-2.3 $(290 \mu \mathrm{m} \times 580 \mu \mathrm{m})$ rectangular waveguide. The IF backend covers at least an instantaneous bandwidth of $17 \mathrm{GHz}$, which extends the bandwidth of current receivers at submillimeter wavelengths by a factor of a few (four factors with respect to the current ALMA receiving system).

\section{Receiver system}

The wideband receiver system is based on three key subsystems: (1) the receiver frontend, (2) the dual-band LO system, and (3) the IF backend (see Fig. 1). The receiver frontend is capable of receiving the RF signal across the $275-500 \mathrm{GHz}$ frequency range and output the down-converted IF signals in the 4-21 GHz range.

The cryostat in our tests uses a vacuum window with a $12.5 \mu \mathrm{m}$ thick polyimide film and an infrared filter for the RF signal input. In the cryostat, a wideband corrugated horn antenna, a WR-2.3 waveguide LO/RF coupler with a coupling ratio of around $15 \mathrm{~dB}$, and the SIS-mixer-preamplifier module are placed on the 4-K stage. The design and performance of the RF components are described in previous papers (Gonzalez et al. 2017a; Kojima et al. 2018). The key component in the receiver frontend is a low-noise heterodyne mixer module to achieve wide RF and IF coverage. The detailed design is summarized in Appendix A. We employed SIS junctions with high critical current density $\left(J_{\mathrm{C}}\right)$ based on $\mathrm{Nb} / \mathrm{Al} / \mathrm{AlN} \mathrm{N}_{x} / \mathrm{Al} / \mathrm{Nb}$ trilayers. The high- $J_{\mathrm{C}}$ junctions yield a lower $R_{\mathrm{N}} C$ product, which facilitates the broadband matching for both RF and IF circuits without the need of trade-off considerations, where $R_{\mathrm{N}}$ and $C$ indicate normal state resistance and capacitance of the SIS junction. In addition, the real part of the junction impedance in the SIS mixer can be flexibly adjusted by selecting the appropriate junction size. Taking advantage of these characteristics of high- $J_{C}$ junctions, we adopted a parallel-connected twin junction (PCTJ) to achieve both wide RF and IF bandwidth. The IF output circuity is designed to take the IF impedance of the SIS junction and the noise parameters and $S$-parameters of the cryogenic low-noise amplifier (CLNA) into account. The CLNA, manufactured by Low Noise Factory ${ }^{2}$, has a typical gain and noise temperature of $35 \mathrm{~dB}$ and $5 \mathrm{~K}$ over 3-22 GHz. It incorporates an input-matching circuit optimized for $50 \Omega$ standard impedance and a bias-tee circuit for the SIS junctions (Kojima et al. 2018).

The dual-band LO injection system employs two LO sources covering the frequency ranges of $283-365 \mathrm{GHz}$ and $393-$ $492 \mathrm{GHz}$, and a waveguide diplexer to combine their outputs into a single waveguide (Gonzalez 2017b). Each LO source is composed of a millimeter-wave high-power source at room temperature followed by a cold multiplier located on the cryostat $10 \mathrm{~K}$

2 http://www. lownoisefactory.com/ 
Table 1. Frequency ranges of the receiver IF and ADC input at each channel.

\begin{tabular}{lcc}
\hline \hline Channel & Receiver IF & ADC input \\
\hline 1 & $4.0-11.5 \mathrm{GHz}$ & $4.0-11.5 \mathrm{GHz}$ \\
2 & $11.3-21.0 \mathrm{GHz}$ & $1.8-11.5 \mathrm{GHz}$ \\
\hline
\end{tabular}

stage. This system allows us to fully cover the $275-500 \mathrm{GHz}$ RF band in a seamless manner.

After the down-conversion and amplification of the signal in the heterodyne module, the IF signal is further amplified with a second CLNA, and then transmitted to the IF backend system at room temperature. The IF backend system defines the receiver IF bandwidth, divides the IF band into two channels for Ch.1: 4.011.5 GHz and Ch.2: $11.3-21.0 \mathrm{GHz}$ (see Table 1), and records the time-dependent signals. The IF chain at room temperature uses a low-pass filter (LPF) $\left(f_{\mathrm{C}}=21 \mathrm{GHz}\right)$ and a third roomtemperature LNA with $3-22 \mathrm{GHz}$ range to minimize the noise contribution from the whole IF chain. The $4-21 \mathrm{GHz}$ IF band is divided into two channels using a power divider, an LPF $\left(f_{\mathrm{C}}=\right.$ $11.5 \mathrm{GHz})$, and high pass filter (HPF) $\left(f_{\mathrm{C}}=10.5 \mathrm{GHz}\right)$. The IF signal at Ch. 2 is further down-converted to $1.8-12.3 \mathrm{GHz}$ with a second LO frequency of $22.8 \mathrm{GHz}$. Then, the frequency band for Ch.2 is defined as $1.8-11.5 \mathrm{GHz}$ with the LPF $\left(f_{\mathrm{C}}=11.5 \mathrm{GHz}\right)$ and finally received by the ADC. Thus, the IF backend provides a $0.2 \mathrm{GHz}$ overlap between the channels at around IF $11.4 \mathrm{GHz}$. The ADC used in this experiment is the M8131A digitizer produced by Keysight technologies (Keysight Technologies 2019). The M8131A has two channels, and each channel is capable of 32 Gsps sampling and $12.5 \mathrm{GHz}$ analog bandwidth with 10 bit resolution. It adopts an interleaving technique that uses two ADCs running at 16 Gsps. Each of the 16 Gsps ADC cores is multiplexed from a number of sub-ADCs that are running at a lower rate and are operated by an internal clock of $8 \mathrm{GHz}$. The effective number of bits (ENOB) of the ADC ensures above 6.5. The signal-to-noise ratio $S / N(\mathrm{~dB})=6.02 E N O B+1.76$, which guarantees a dynamic range of more than $40 \mathrm{~dB}$ with respect to quantization noise. The digitized raw samples can be stored in an internal acquisition memory with $2 \mathrm{~GB}$ or streamed over four optical data interface (ODI) with 160 Giga bits per second for each. The streaming capability using the ODI offers real-time signal processing, which is required for the correlator system in an interferometer. However, in this experiment, the internal memory was used and then the recorded data was processed using an external computer. Note that the M8131A for this experiment is a protype demonstrator under development, and therefore performances may not meet the product specification. Thus, the ADCs fully digitize the final IF band for both channels, and the receiver system developed here enables an instantaneous bandwidth of $17 \mathrm{GHz}$.

\section{Wideband experiment}

We characterized the heterodyne receiver using two types of RF signals using a noise source and comb source. First, the noise temperature measurement was conducted based on the standard $Y$-factor method with room temperature $(295 \mathrm{~K})$ and liquid-nitrogen $(77 \mathrm{~K})$ blackbody loads. In order to characterize the performance of the receiver frontend, the noise temperature was measured at the output of the third IF amplifier using a spectrum analyzer (SPA) and power meter. Then, the noise temperature of the receiver full system including the IF backend was compared between the SPA and the ADC for each channel. Second, we performed the heterodyne experiment by means of the ADC and the RF comb source. The RF comb signal source consists of a microwave comb generator and a frequency up-converter. The microwave comb generator uses a step-recovery diode and a synthesizer with a reference signal of $100 \mathrm{MHz}$ (Kiuchi et al. 1999). It outputs base-band comb signals ranging from $100 \mathrm{MHz}$ up to $25 \mathrm{GHz}$ with a $100 \mathrm{MHz}$ spacing. The RF comb signal is generated by up-converting the base-band comb signals with a commercially available WR-3.4 $(432 \mu \mathrm{m} \times 864 \mu \mathrm{m})$ waveguide harmonic mixer and LO power. This combination of using the ADC and RF comb source allows us to effectively verify the heterodyne receiver in a very short time, compared to the case of sweeping single-tone CW signals.

The inset in the graph in Fig. 2 (left) shows the measured current voltage $(I-V)$ characteristics of an $\mathrm{Nb} / \mathrm{Al} / \mathrm{AlN}{ }_{x} / \mathrm{Al} / \mathrm{Nb}-$ based twin junction, fabricated at the National Astronomical Observatory of Japan. The critical current density $J_{\mathrm{C}}$ and subgap-to-normal state resistance ratio $R_{\mathrm{sg}} / R_{\mathrm{N}}$ of the twin junction were $31 \mathrm{kA} \mathrm{cm}^{-2}$ and 15 . The figure also presents the $I-V$ curve pumped by LO power at $355 \mathrm{GHz}$ and the total IF output power for the blackbody sources measured with the power meter. DSB noise temperature averaged over the IF band as a function of LO frequency is shown in Fig. 2 (left). The result shows a noise temperature as low as two to three times the quantum noise $\left(h f / k_{\mathrm{B}}\right)$ across the entire LO frequency range. Figure 2 (right) shows the noise temperature and IF output power of the receiver frontend for $295 \mathrm{~K}$ blackbody radiation as a function of IF frequency, measured with the SPA and at the LO frequencies of 315,400 , and $460 \mathrm{GHz}$. The IF output power is recorded under the condition in a resolution bandwidth of $8 \mathrm{MHz}$ and video bandwidth of $100 \mathrm{~Hz}$ and is averaged over three measurements. It takes $33 \mathrm{~s}$ per single sweep across this IF range. The receiver IF band can be defined by roughly $4-21 \mathrm{GHz}$ from the measured IF power. A relatively large peak-to-peak gain slope of around $9 \mathrm{~dB}$ was measured, which comes from the SIS-mixerpreamplifier module because of the impedance-matching conditions between the SIS junctions and the monolithic microwave integrated circuit (MMIC) in the CLNA. The measured noise temperature shows reasonable flatness and good performance across the full 4-21 GHz IF band. The noise ripple features agree well with simulations, as discribed in Appendix A. The noise increment at around IF $10,15.5$ and $21 \mathrm{GHz}$, and the LO frequency of $460 \mathrm{GHz}$ is slightly higher than measurements at the lower LO frequencies. This is a result of the higher IF noise contribution to the receiver noise temperature, which is due to the lower mixer gain at the higher end of the LO frequency range. However, overall noise performance is comparable with modern SIS receivers with much narrower IF bands, such as 4-8 GHz. These results indicate that the full receiver frontend system, including both the RF and IF components developed here and the LO chain, works as expected. This is the first demonstration of a low-noise receiver frontend with wide RF ( $\sim 60 \%$ fractional bandwidth) and wide IF (4-21 GHz) performance.

Figure 3 shows the comparison of noise temperature at the LO frequency of $355 \mathrm{GHz}$, which is recorded with the SPA and ADC at each channel. For the ADC, the time-dependent voltage was recorded for a total of $256 \mu$ s with the sampling interval of 31.25 ps (32 Gsps) as a single data set because the internal memory in the ADC module is limited. A total of 16 data sets were individually acquired and combined as a single data set with a length of $4.1 \mathrm{~ms}$ in total. Afterward, the $4.1 \mathrm{~ms}$ data set was divided into a total of 32000 time frames with a length of $128 \mathrm{~ns}$ each. Fast Fourier transforms were applied to each of 

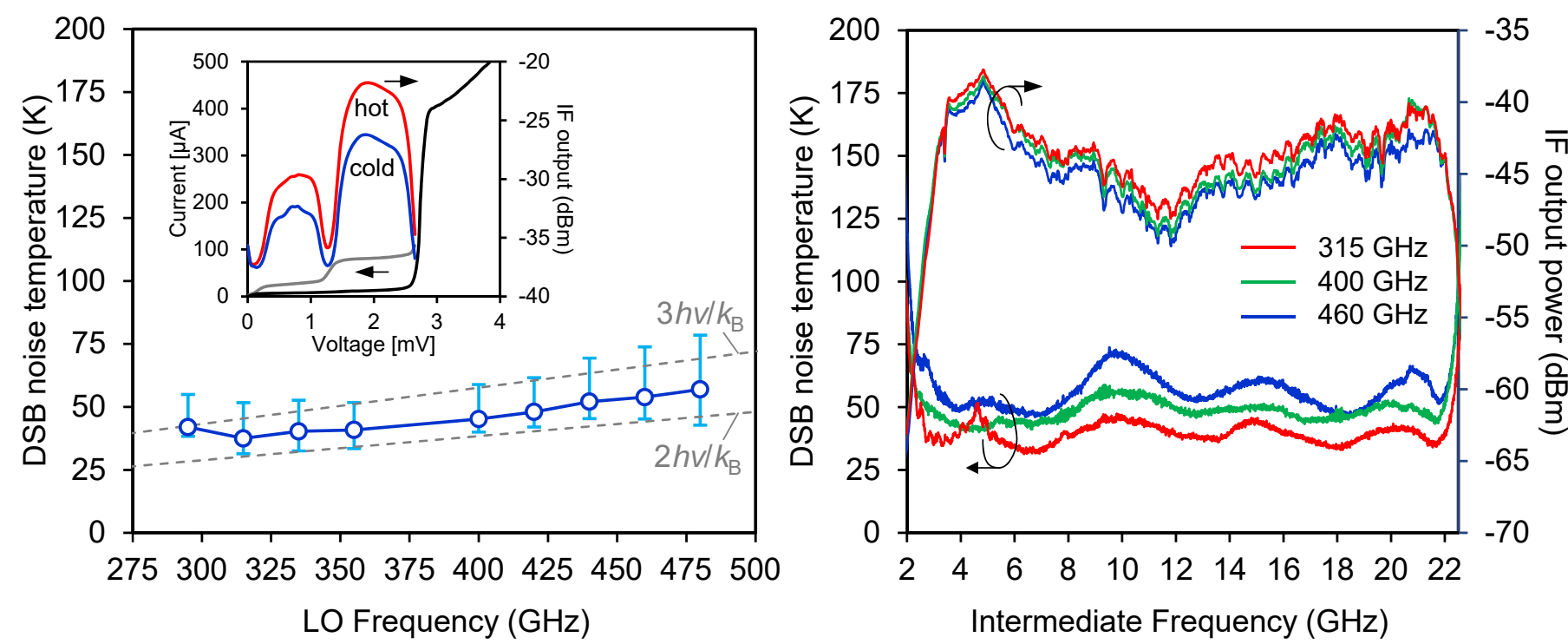

Fig. 2. Left: DSB receiver noise temperature measured with a power meter as a function of LO frequency. The inset indicates the current-voltage characteristics and IF output power responses for hot (red) and cold (blue) blackbody radiations. Vertical bars indicate the minimum and maximum noise temperatures across the IF frequency range based on the measurements from the SPA. Right: DSB noise temperature and IF output power for the hot load recorded with the SPA at LO frequencies of 315,400 , and $460 \mathrm{GHz}$ as a function of IF.

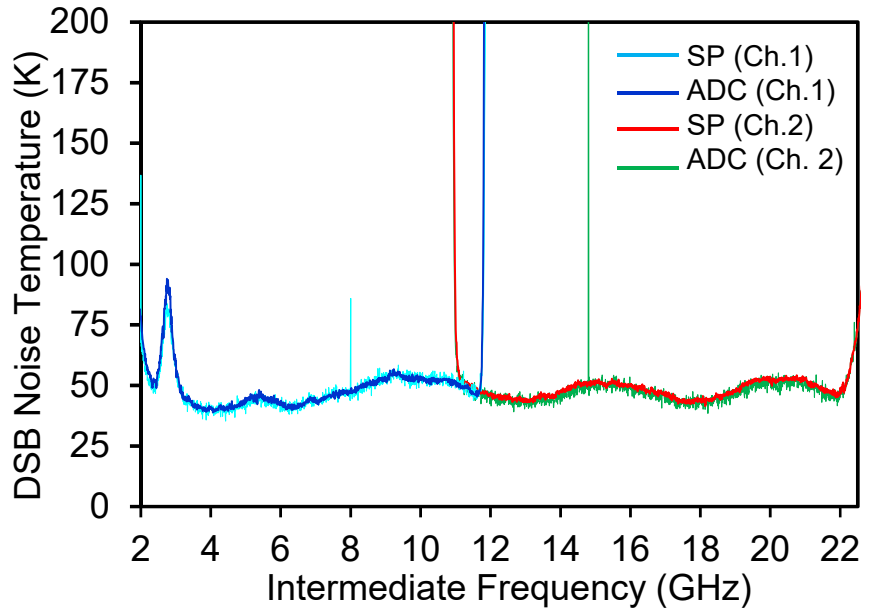

Fig. 3. Comparison of receiver noise temperature measured at the LO frequency of $355 \mathrm{GHz}$ with the SPA and the ADC. The peaks at $8.0 \mathrm{GHz}$ and $14.8 \mathrm{GHz}$ correspond to the frequency of the sampling clock.

them, and then, the 32000 frequency-domain sets were averaged. This procedure improves the $\mathrm{S} / \mathrm{N}$ in the derived noise temperature, but reduces the frequency resolution. The noise temperatures for both channels are represented as a function of IF frequency in Fig. 3. The peaks at IF $8.0 \mathrm{GHz}$ and $14.8 \mathrm{GHz}$ are contamination from the $8 \mathrm{GHz}$ sampling clock that is internally provided in the ADC. The noise temperatures measured between the SPA and ADC agreed well. In addition, the noise temperature measured with both the SPA and ADC are smoothly connected at the cross-over frequencies. These measurements and their comparison were conducted at each LO frequency. These results guarantee the validity of the noise temperature measurement using the ADC within the IF power range and ripple levels provided by the receiver system frontend and analog IF circuitry.

We further characterized the full receiver system by means of the ADC and an RF comb source at around $290 \mathrm{GHz}$. For the harmonic mixer, we selected the harmonic number of 10 and an LO frequency of $29 \mathrm{GHz}$. Thus, the RF comb signal can be widely distributed over $265-315 \mathrm{GHz}$ band with the $100 \mathrm{MHz}$ spacing. The receiver was set at the LO frequency of $315 \mathrm{GHz}$, and thus the RF comb signal mostly spread in the lower sideband for the receiver LO frequency. The RF comb signal may exist on the upper sideband of the receiver because no LPF was used in the microwave comb signal. However, these signals are just received as a DSB signal. Figure 4a represents the amplitude of the comb signal analyzed for the time frame of $256 \mu \mathrm{s}$, which corresponds to a frequency resolution of $3.9 \mathrm{kHz}$. The measured IF spectrum is corrected for the receiver baseline with the following procedure. The IF signal received at the ADC is given by

$P_{\mathrm{IF}}=G_{\mathrm{rx}}\left(P_{\mathrm{sig}}+P_{\mathrm{hot}}+P_{\mathrm{rx}}\right)$,

where $P_{\text {sig }}, P_{\text {hot }}, P_{\mathrm{rx}}$, and $G_{\mathrm{rx}}$ indicate the input power of the RF comb signal, the radiation power from the blackbody load, the equivalent receiver noise power, and the receiver gain, respectively. Thus, the input power $P_{\text {sig }}$ can be estimated by correcting for all the other parameters, which are recorded through the $Y$-factor measurement. The comb signal spreads across the wide IF range, and the frequency spacing of the comb signal is precisely $100 \mathrm{MHz}$. Figures $4 \mathrm{~b}$ and $\mathrm{c}$ indicate the portion of the two IF signals around the cross-over frequency and the enlarged view of the IF signal at $11.4 \mathrm{GHz}$, respectively. The IF signal at $11.4 \mathrm{GHz}$ shows good consistency in the two channels with respect to amplitude and frequency. The root-mean-square amplitude difference between two channels is $0.8 \mathrm{~dB}$ in the $11.4 \pm 0.3 \mathrm{GHz}$ range. The IF signals received at both channels in the cross-over frequency range must originate from the same RF signal. Thus, these results secure the connection between two channels. This verification cannot be made using a noise source because it is difficult to identify the exact frequencies. This method based on the RF comb source can also be extended to verify the phase connectivity between the channels, which must be confirmed for interferometric observations, such as with ALMA. 


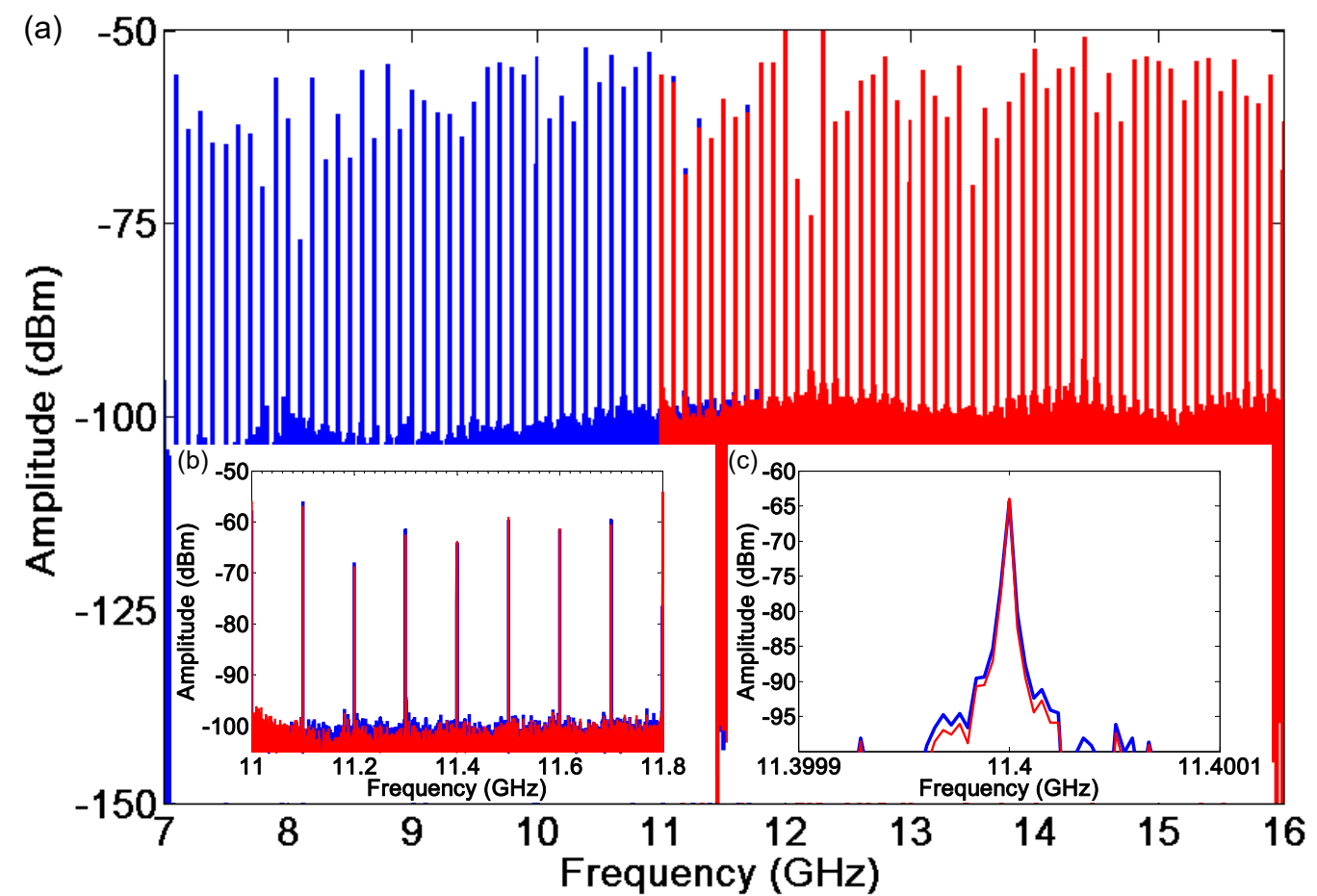

Fig. 4. Analyzed result of IF output signals for the broadband RF comb signal injection with a $100 \mathrm{MHz}$ spacing at cross-over frequencies around 11.4 GHz. The LO frequency for the receiver frontend is $315 \mathrm{GHz}$.

\section{Conclusions}

We have reported on a $275-500 \mathrm{GHz}$ wide RF full receiver system with an instantaneous bandwidth of $17 \mathrm{GHz}$. The receiver noise temperature was below three times the quantum noise across the RF range. The characterization of the full receiver system using an ADC and RF comb source guarantees general heterodyne operation and the connectivity between two channels. The wideband low-noise receiver and associated technologies demonstrated in this study are ready to be applied for next-generation instrumentation for millimeter- and submillimeter-wave telescopes.

Acknowledgements. The authors would like to express their gratitude to J. Horinaga and K. Uto of Keysight technologies for their support in the use of the M8131A digitizer. This work was supported in part by the Japan Society for the Promotion of Science KAKENHI under Grant $15 \mathrm{H} 02074$.

\section{References}

ALMA Development Working Group, Carpenter, J., Iono, D., et al. 2018, ALMA Memo Series, 612, http://library.nrao.edu/public/memos/ alma/main/memo612.pdf

Baryshev, A. M., Hesper, R., Mena, F. P., et al. 2015, A\&;A, 577, A129
Billade, B., Pavolotsky, A., \& Belitsky, V. 2013, IEEE Trans. Terahertz Sci. Technol., 3, 416

Gonzalez, A., Kaneko, K., Asayama, S., et al. 2017a, Proc. 11th Eur. Conf. IEEE Antennas Propag., 16900258

Gonzalez, A., Kojima, T., Kaneko, K., et al. 2017b, IEEE Trans. Terahertz Sci. Technol., 7, 6

Mahieu, S., Maier, D., Lazareff, B., et al. 2012, IEEE Trans. Terahertz Sci. Technol., 2, 1

Novoselov, E., \& Cherednichenko, S. 2017, Appl. Phys. Lett., 110, 032601 Kerr, A. R., Pan, S.-K., et al. 2014, IEEE Trans. Terahertz Sci. Technol., 4, 2

Keysight Technologies 2019, M8131A-16/32 GSa/s Digitizer Preliminary Data Sheet, version 0.8 , https://www.keysight.com/us/en/assets/ 7018-06368/data-sheets/5992-3412.pdf

Kiuchi, H., Takahashi, Y., Kurihara, N., et al. 1999, IEEE Trans. Instr. Meas., 48, 6

Kojima, T., Kroug, M., Uemizu, K., et al. 2017, IEEE Trans. Terahertz Sci. Technol., 7, 6

Kojima, T., Kroug, M., Uemizu, K., et al. 2018, IEEE Trans. Terahertz Sci. Technol., 8, 638

Kooi, J. W., Sumner, M. C., Ceria, R., et al. 2007, IEEE Trans. Microw. Theory Techn., 55, 10

Tamura, T., Arayashiki, T., Oyama, M., et al. 2015, IEEE Trans. Appl. Supercond., 25, 3

Tong, C. E., Grimes, P. K., Weintroub, J., et al. 2015, IEEE MTT-S International Microwave Symposium

Uzawa, Y., Fujii, Y., Gonzalez, A., et al. 2015, IEEE Trans. Appl. Supercond., 25,3 


\section{Appendix A: SIS mixer design}

The SIS-mixer-preamplifier module used in this study is composed of four parts: (1) the main metal body, which provides support for the other parts and a WR-2.3 waveguide interface for the RF signal input, (2) a superconducting magnetic coil, (3) a dropin-type CLNA with a 4-21 GHz IF output, and (4) the SIS mixer chip. The basic design concepts of the RF and IF circuitries are similar to the design previously described in Kojima et al. (2018) and Kojima et al. (2017), respectively, while the IF-matching circuit in the SIS mixer chip is optimized to maximize the IF bandwidth of the module. The SIS mixer chip is mounted onto the mixer chip slot inside the main body, and is directly connected with the CLNA by bonding wires. It contains a singleside waveguide probe to couple the signal coming from the main body RF input to the chip, and integrates an RF matching circuit, SIS junctions, and IF output circuity, as shown in Fig. A.1 (top). We adopted a PCTJ employing high- $J_{\mathrm{C}}$ SIS junctions based on $\mathrm{Nb} / \mathrm{Al} / \mathrm{AlN}_{x} / \mathrm{Al} / \mathrm{Nb}$ trilayers to achieve both wide $\mathrm{RF}$ and IF bandwidth.

In this design, we assume that the RF resistance $R_{\mathrm{RF}} \sim$ $R_{\mathrm{N}} / 2=4.3 \Omega$ and the IF resistance $R_{\mathrm{IF}} \sim a R_{\mathrm{N}} / 2(8<a<$ 16) for the SIS junction that has a diameter of $0.9 \mu \mathrm{m}$ and $J_{\mathrm{C}}=40 \mathrm{kA} \mathrm{cm}^{-2}$. The RF probe is designed to have impedance around $35 \Omega$ over the full $275-500 \mathrm{GHz}$ range, and a two-section impedance transformer made of $\mathrm{Nb} / \mathrm{SiO}_{2} / \mathrm{Nb}(200 / 400 / 650 \mathrm{~nm})$ microstrip lines between the RF probe and the PCTJ. The variation in the IF resistance $R_{\mathrm{IF}}$, from 33 to $67 \Omega$, is empirically given by the LO frequency-dependent dynamic resistance of the SIS junction. The IF output circuity is designed taking the IF impedance of the SIS junction and the noise parameters and $S$-parameters of the CLNA into account. It consists of an LPF section, a capacitor implemented by a low characteristic impedance line, and a bonding pad. The LPF is designed to suppress the RF signal leakage and to output the IF signal with low loss. The geometric topology of the LPF uses a combination of alternating microstrip and coplanar waveguides as low $(\sim 20 \Omega)$ and high $(\sim 90 \Omega)$ characteristic impedance lines, respectively. The capacitor is placed at the end of the LPF section. In this configuration, the LPF and the capacitor form a П-type network in combination with the parasitic capacitances associated with the RF matching circuit, including the SIS junction and the microstrip line (Kooi et al. 2007). The area of the capacitor was adjusted to optimize the IF performance in terms of noise temperature and bandwidth.

Figure A.1 (bottom) shows the result of a simplified calculation of the receiver noise temperature for the designed IF circuit. The CLNA performance for the IF resistance $R_{\mathrm{IF}}$ (33$67 \Omega$ ) is just calculated on the basis of the designed IF circuit and the CLNA $S$-parameters and noise parameters. We assumed DSB mixer gain of $0 \mathrm{~dB}$ and mixer noise temperature of $38 \mathrm{~K}$ $\left(=2 h f / k_{\mathrm{B}}\right.$ at $\left.400 \mathrm{GHz}\right)$, which are constant to the variation of the IF resistance $R_{\mathrm{IF}}$. The calculated receiver noise temperature is approximately $50 \mathrm{~K}$ on average over $4-21 \mathrm{GHz}$ and with a ripple of $\pm 8 \mathrm{~K}$. This ripple appears because of the noise-matching conditions between the SIS junctions and the MMIC in the
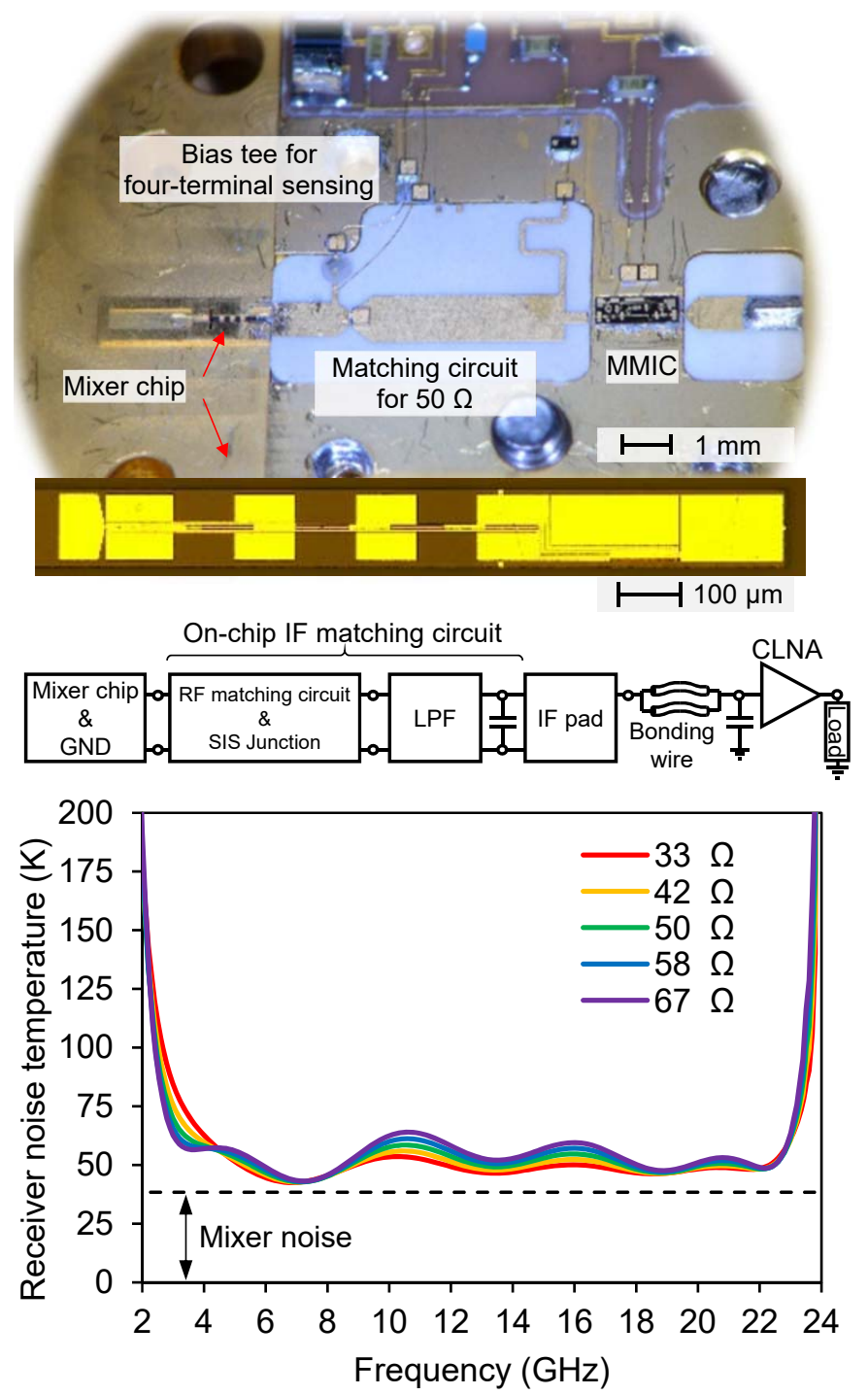

Fig. A.1. Top: photograph of the mixer chip and inside of the SIS-mixerpreamplifier module. Bottom: schematic of the equivalent circuit used in this calculation and calculated receiver noise temperature for different IF resistance values of the SIS junctions.

CLNA. The DSB noise temperature of about $50 \mathrm{~K}$ is comparable with the current receiver performance in this frequency range and with $4-8 \mathrm{GHz}$ IF bandwidth, such as the ALMA band 8 (RF: 385-500 GHz) receiver (Tamura et al. 2015). Therefore, a significant continuum sensitivity improvement by the larger bandwidth can be expected without degrading spectral line sensitivity. The averaged noise temperature deteriorates as the IF resistance increases, while the IF bandwidth becomes slightly wider at the lower frequency. However, because higher IF resistance yields larger mixer gain, the additional gain reduces the effect of the additional noise in the overall receiver noise temperature. 